Pacific Journal of Mathematics

QUASINORMAL STRUCTURES FOR CERTAIN SPACES O 


\title{
QUASI-NORMAL STRUCTURES FOR CERTAIN SPACES OF OPERATORS ON A HILBERT SPACE
}

\author{
Anthony To-Ming LaU and Peter F. Mah
}

\begin{abstract}
Let $E$ be a dual Banach space. $E$ is said to have quasi-weak*-normal structure if for each weak* compact convex subset $K$ of $E$ there exists $x \in K$ such that $\|x-y\|<\operatorname{diam}(K)$ for all $y \in K$. $E$ is said to satisfy Lim's condition if whenever $\left\{x_{\alpha}\right\}$ is a bounded net in $E$ converging to 0 in the weak* topology and $\lim \left\|x_{\alpha}\right\|=s$ then $\lim _{\alpha}\left\|x_{\alpha}+y\right\|=s+\|y\|$ for any $y \in E$. Lim's condition implies (quasi) weak*-normal structure. Let $H$ be a Hilbert space. In this paper, we prove that $\mathscr{T}(H)$, the space of trace class operators on $H$, always has quasi-weak*-normal structure for any $H ; \mathscr{T}(H)$ satisfies Lim's condition if and only if $H$ is finite dimensional. We also prove that the space of bounded linear operator on $H$ has quasi-weak*-normal structure if and only if $H$ is finite dimensional; the space of compact operators on $H$ has quasi-weak-normal structure if and only if $H$ is separable. Finally we prove that if $X$ is a locally compact Hausdorff space, then $C_{0}(X)^{*}$ satisfies Lim's condition if and only if $C_{0}(X)^{*}$ is isometrically isomorphic to $l_{1}(\Gamma)$ for some non-empty set $\Gamma$.
\end{abstract}

1. Introduction. Let $E$ be a Banach space. A bounded convex subset $K$ of $E$ has normal structure if every non-trivial convex subset $H$ of $K$ contains a point $x_{0}$ such that

$$
\sup \left\{\left\|x_{0}-y\right\|: y \in H\right\}<\operatorname{diam}(H) .
$$

Here $\operatorname{diam}(H)=\sup \{\|x-y\|: x, y \in H\}$ denotes the diameter of $H$. The Banach space $E$ is said to have normal structure if every bounded closed convex subset of $E$ has normal structure. If $E$ is a dual space then $E$ is said to have weak* normal structure if every weak* compact convex subset of $E$ has normal structure. In [6] Lim introduced the notion of weak* normal structure and proved that $l_{1}$ has this property. It also follows from the proof of Theorem 3 in [4] that $l_{1}(\Gamma)$ has the same property for any non-empty set $\Gamma$. Furthermore, an application of Proposition 2 in [9] shows that $l_{\infty}(\Gamma)$ has weak* normal structure if and only if $\Gamma$ is a finite set.

Let $H$ be a Hilbert space. Let $\mathscr{B}(H)$ be the space of bounded linear operators from $H$ into itself with the operator norm. Let $\mathscr{C}(H)$ be the closed ideal of compact operators in $\mathscr{B}(H)$. Then, as is well known, 
$\mathscr{C}(H)^{* *}=\mathscr{B}(H)$ and $\mathscr{C}(H)^{*}$ can be identified with $\mathscr{T}(H)$, the trace-class operators on $H$ with the trace norm (see [12, pp. 63-64]). When $H$ is infinite dimensional, it is known that $\mathscr{C}(H)$ and $\mathscr{T}(H)$ contain isometric copies of $c_{0}$ and $l_{1}$, respectively [12, Proposition 1.4 and Theorem $1.6 \mathrm{p}$. 62]. It follows, then, that the Banach spaces $\mathscr{C}(H), \mathscr{T}(H)$ and $\mathscr{B}(H)$ do not have normal structure unless $H$ is finite dimensional [1].

A concept weaker than that of normal structure was introduced by Soardi in [11]. A bounded convex subset $K$ of a Banach space has quasi-normal structure (or close-to-normal structure [13]) if for every non-trivial closed convex subset $H$ of $K$, there exists $x \in H$ such that $\|x-y\|<\operatorname{diam}(H)$ for all $y \in H$. A Banach space has quasi-normal structure (quasi-weak-normal structure) if every bounded (weakly compact) closed convex subset has quasi-normal structure. If, in addition, it is a dual Banach space then it has quasi-weak*-normal structure if every weak* compact convex subset has quasi-normal structure.

In $\$ 2$ of this paper, we prove, among other things, three theorems on quasi-normal structure and its generalizations for certain spaces of operators on a Hilbert space $H$. First, we prove that $\mathscr{B}(H)$ has quasi-weak*normal structure if and only if $H$ is finite dimensional (Theorem 1). Secondly, we prove that $\mathscr{T}(H)$ has quasi-weak*-normal structure for any $H$ (Theorem 2). Finally, we prove that $\mathscr{C}(H)$ has quasi-weak-normal structure if and only if $H$ is separable (Theorem 3). A table summarizing our results is provided at the end.

Let $E$ be a Banach space. Then $E^{*}$ is said to satisfy Lim's condition if whenever $\left\{\phi_{\alpha}\right\}$ is a bounded net in $E^{*}, \phi_{\alpha}$ converges to 0 in the weak* topology and $\lim _{\alpha}\left\|\phi_{\alpha}\right\|=s$, then $\lim _{\alpha}\left\|\phi_{\alpha}+\psi\right\|=s+\|\psi\|$ for any $\psi \in E^{*}$. In [6], Lim showed that $l_{1}$ satisfies this condition for sequences. Also a simple modification of the proof of Theorem 3 [4] shows that Lim's condition implies weak* normal structure (see Lemma 4). We prove in section 4 that (Theorem 4 ) if $X$ is a locally compact Hausdorff space, then the dual Banach space $C_{0}(X)^{*}$ satisfy Lim's condition if and only if $C_{0}(X) *$ is isometric isomorphic to $l_{1}(\Gamma)$ for some non-empty set $\Gamma$. We also prove that (Theorem 5) if $H$ is a Hilbert space, then $\mathscr{T}(H)$ satisfy Lim's condition if and only if $H$ is finite dimensional.

As known [6, Theorem 1], if $E$ is dual Banach space with weak* normal structure, then every nonexpansive mapping $T$ of a non-empty weak* compact convex subset $K$ of $E$ (i.e. $\|T x-T y\| \leq\|x-y\|$ for any $x, y \in K$ ) into itself has a fixed point. Also [13, Theorem 1] if $E$ is a Banach space with quasi-weak-normal structure if and only if every 
Kannan map $T$ of a non-empty weakly compact convex subset $K$ of $E$ (i.e. $\|T x-T y\| \leq(\|x-T x\|+\|y-T y\|) / 2$, for any $x, y \in K)$ into itself has a fixed point.

\section{Quasi-normal structures.}

THEOREM 1. Let $H$ be a Hilbert space. Then $\mathscr{B}(H)$ has quasi-weak*normal structure if and only if $H$ is finite dimensional.

Proof. If $H$ is finite dimensional, then $\mathscr{B}(H)$ is finite dimensional. Hence $\mathscr{B}(H)$ has normal structure.

Conversely if $H$ is infinite dimensional, write $H=l_{2}(\Gamma)$ where $\Gamma$ is a complete orthonormal basis of $H$. Consider the map $\rho: l_{\infty}(\Gamma) \rightarrow \mathscr{B}\left(l_{2}(\Gamma)\right)$ defined by

$$
\rho(f)(h)(t)=f(t) h(t), \quad t \in \Gamma .
$$

Then $\rho$ is an isometry and algebra isomorphism of $l_{\infty}(\Gamma)$ into $\mathscr{B}\left(l_{2}(\Gamma)\right)$ which is continuous when $l_{\infty}(\Gamma)$ has the weak* topology and $\mathscr{B}(H)$ has the weak operator topology. By Proposition 2 in [9], there exists a weak* compact convex subset $K$ of $l_{\infty}(\Gamma)$ such that for each $f \in K$, there exists $g \in K$ with $\|f-g\|_{\infty}=\operatorname{diam}(K)>0$. Since weak* topology and the weak operator topology agree on bounded subset of $\mathscr{B}(H), \rho(K)$ is also a weak* compact convex subset of $\mathscr{B}(H)$ with positive diameter. In particu$\operatorname{lar} \mathscr{B}(H)$ does not have the quasi-weak*-normal structure.

LEMMA 1. Let E be a dual Banach space. Then $E$ has quasi-weak*-normal structure if it satisfies

whenever $\left\{x_{\alpha}\right\}$ is a net in $E, x_{\alpha}$ converges to $x$ in the $(* *) \quad$ weak $^{*}$ topology and $\left\|x_{\alpha}\right\|$ converges to $\|x\|$, then $x_{\alpha}$ converges to $x$ in norm.

Proof. Suppose there exists a weak* compact convex subset $K$ of $E$, $\operatorname{diam}(K)>0$, such that for each $x \in K$, there exists $T(x) \in K$ with $\|x-T(x)\|=\operatorname{diam}(K)$. Following an idea of Wong [13, Theorem 2], let $W(K)$ denote the supremum of $\{|H| ; H$ is a diametral subset of $K\}$ ( $H$ is diametral if $\left\|x_{1}-x_{2}\right\|=\operatorname{diam}(K)$ whenever $\left.x_{1}, x_{2} \in H, x_{1} \neq x_{2}\right)$. As shown in the proof of Theorem 2 in [13], $W(K)$ is infinite. Let $\left\{x_{n}\right\}$ be a sequence in $K$ such that $\left\|x_{n}-x_{m}\right\|=\operatorname{diam}(K), n \neq m$. Since $K$ is weak* compact, there exists a subnet $\left\{x_{n_{\alpha}}\right\}$ of $\left\{x_{n}\right\}$ such that $x_{n_{\alpha}}$ converges to some $z \in K$ in the weak*-topology. Passing to a subnet if necessary, we 
may assume that the net $\left\{\left\|x_{n_{\alpha}}-T(z)\right\|\right\}$ also converges. Then

$$
\operatorname{diam}(K)=\|z-T(z)\| \leq \lim _{\alpha}\left\|x_{n_{\alpha}}-T(z)\right\| \leq \operatorname{diam}(K) .
$$

So $\lim _{\alpha}\left\|x_{n_{\alpha}}-T(z)\right\|=\operatorname{diam}(K)$. Since $\left\{x_{n_{\alpha}}-T(z)\right\}$ converges in the weak* topology to $z-T(z)$, and $\lim _{\alpha}\left\|x_{n_{\alpha}}-T(z)\right\|=\|z-T(z)\|$, it follows that $\left\{x_{n_{\alpha}}-T(z)\right\}$ converges in norm to $z-T(z)$. In particular, the net $\left\{x_{n_{\alpha}}\right\}$ converges in norm to $z$ also. This contradicts the choice of the sequence $\left\{x_{n}\right\}$.

The next lemma is due to K. McKennon [7, Lemma, 3.2]. For the sake of completeness, we give a short proof.

LeMma 2 (McKennon [7]). Let $A$ be a $C^{*}$-algebra and $\left\{e_{\alpha}\right\}$ be an approximate identity of $A, e_{\alpha} \geq 0$ and $\left\|e_{\alpha}\right\| \leq 1$. Let $\left\{\phi_{\beta}\right\}$ be a net in $A^{*}$ such that $\phi_{\beta} \rightarrow \phi$ in the weak ${ }^{*}$ topology and $\left\|\phi_{\beta}\right\| \rightarrow\|\phi\|$. Then for any $\varepsilon>0$, there exists $\alpha_{0}, \beta_{0}$ such that

$$
\left\|R_{e_{\alpha_{0}}} \phi-\phi\right\|<\varepsilon
$$

and

$$
\left\|R_{e_{\alpha_{0}}} \phi_{\beta}-\phi_{\beta}\right\|<\varepsilon
$$

for all $\beta \geq \beta_{0}$, where $R_{e} \phi(x)=\phi(x e)$.

Proof. Let $x \in A,\|x\| \leq 1$. Then using [5, Lemma 3.3] and some properties of positive linear functionals, we obtain the following estimate

$$
\begin{aligned}
\left|\left\langle R_{e_{\alpha}} \phi-\phi, x\right\rangle\right|^{2} & =\left|\left\langle\phi, x \cdot e_{\alpha}-x\right\rangle\right|^{2}=\left|\left\langle\phi, x \cdot\left(1-e_{\alpha}\right)\right\rangle\right|^{2} \\
& \leq\|\phi\||\phi|\left[\left(x \cdot\left(1-e_{\alpha}\right)\right)^{*}\left(x \cdot\left(1-e_{\alpha}\right)\right)\right] \\
& =\|\phi\||\phi|\left[\left(1-e_{\alpha}\right) x^{*} x\left(1-e_{\alpha}\right)\right] \\
& \leq\|\phi\||\phi|\left[\left(1-e_{\alpha}\right)\left(1-e_{\alpha}\right)\right] \leq\|\phi\||\phi|\left(1-e_{\alpha}\right),
\end{aligned}
$$

where 1 is the identity in the enveloping von Neumann algebra $A^{* *}$ of $A$ and $|\phi|$ is the absolute value of $\phi$. Since the net $\left\{e_{\alpha}\right\}$ converges to 1 in the weak* topology of $A^{* *},(1)$ follows from the above estimate.

A similar estimate as above shows that

$$
\left\|R_{e_{\alpha_{0}}} \phi_{\beta}-\phi_{\beta}\right\| \leq\left\|\phi_{\beta}\right\|\left|\phi_{\beta}\right|\left(1-e_{\alpha_{0}}\right) \text {. }
$$

Using [5, Lemma 3.5] and the fact that for each positive form $\left|\phi_{\beta}\right|$, $\left\|\left|\phi_{\beta}\right|\right\|=\left\|\phi_{\beta}\right\|=\left|\phi_{\beta}\right|(1)$, the right side of the above estimate converges to $\|\phi\||\phi|\left(1-\mathrm{e}_{\alpha_{0}}\right)$. Hence (2) follows. 
TheOREM 2. Let $H$ be a Hilbert space. Then $\mathscr{T}(H)$ has the quasiweak*-normal structure.

Proof. By Lemma 1, it suffices to show that $\mathscr{T}(H)=\mathscr{C}(H)^{*}$ has property $(* *)$. Let $\mathscr{P}$ denote all orthogonal projections of $H$ onto a finite dimensional subspace of $H$. Order $\mathscr{P}$ by: $P \geq Q$ iff $Q P=P Q=Q$. Then $(\mathscr{P}, \leq)$ is an approximate identity for $\mathscr{C}(H)$. Since every $T \in \mathscr{C}(H)$ can be written in the form $T=T_{1}+i T_{2}, T_{1}$ self-adjoint, $i=1,2$, it suffices to show that if $T$ is self-adjoint, then $\lim \|T P-T\|=\lim \|P T-T\|=0$. Indeed, if $T \in \mathscr{C}(H)$ and $T$ is self-adjoint, then by the spectral theorem $T=\sum_{i=1}^{\infty} \lambda_{l} P_{i}$, where $\lambda_{i} \rightarrow 0$ as $i \rightarrow \infty$ and $P_{l} \in \mathscr{P}$. Given $\varepsilon>0$ choose $n$ such that $\left\|T-\sum_{i=1}^{n} \lambda_{i} P_{l}\right\|<\varepsilon$. Let $Q \in \mathscr{P}$ be such that $Q \geq P_{i}, \quad i=$ $1,2, \ldots, n$. Then for all $P \geq Q$,

$$
\|T P-T\| \leq\left\|T P-S_{n} P\right\|+\left\|S_{n} P-S_{n}\right\|+\left\|S_{n}-T\right\|<2 \varepsilon,
$$

where $S_{n}=\sum_{i=1}^{n} \lambda_{l} P_{l}$. Similarly, we can show $\lim \|P T-T\|=0$. We also note that each $P \in \mathscr{P}$ is positive and has norm one.

Let $\left\{\phi_{\beta}\right\}$ be a net in $\mathscr{C}(H)^{*}$ converging to some $\phi \in \mathscr{C}(H)^{*}$ in the weak* topology and $\left\|\phi_{\beta}\right\| \rightarrow\|\phi\|$. By Lemma 2 , there exists $P_{0} \in \mathscr{P}$ and $\beta_{0}$ such that

$$
\left\|R_{P_{0}} \phi-\phi\right\|<\varepsilon / 2 \text { and }\left\|R_{P_{0}} \phi_{\beta}-\phi_{\beta}\right\|<\varepsilon / 2
$$

for all $\beta \geq \beta_{0}$. By considering the reversed $C^{*}$-algebra, we may also assume that

$$
\left\|L_{P_{0}} \phi-\phi\right\|<\varepsilon / 2 \text { and }\left\|L_{P_{0}} \phi_{\beta}-\phi_{\beta}\right\|<\varepsilon / 2 .
$$

where $\left(L_{P_{0}} \phi\right)(T)=\phi\left(P_{0} T\right), T \in \mathscr{C}(H)$. Consequently, if $\beta \geq \beta_{0}$,

$$
\left\|R_{P_{0}} L_{P_{0}} \phi-\phi\right\| \leq\left\|R_{P_{0}} L_{P_{0}} \phi-R_{P_{0}} \phi\right\|+\left\|R_{P_{0}} \phi-\phi\right\|<\varepsilon
$$

since $\left\|R_{P_{0}}\right\| \leq\left\|P_{0}\right\|=1$ by (3) and (4). Similarly

$$
\left\|R_{P_{0}} L_{P_{0}} \phi_{\beta}-\phi_{\beta}\right\|<\varepsilon .
$$

Also, $P_{0} \mathscr{C}(H) P_{0}$ is a finite dimensional algebra over $C$. Hence, $\left\{\phi_{\beta}\right\}$, restricted to $P_{0} \mathscr{C}(H) P_{0}$ converges to $\phi$ in norm. Consequently, there exists $\beta_{1} \geq \beta_{0}$ such that

$$
\left\|T_{P_{0}} L_{P_{0}} \phi_{\beta}-R_{P_{0}} L_{P_{0}} \phi\right\|<\varepsilon
$$

if $\beta \geq \beta_{1}$. Now if $\beta \geq \beta_{1}$, we have

$$
\begin{aligned}
\left\|\phi_{\beta}-\phi\right\| \leq & \left\|\phi_{\beta}-R_{P_{0}} L_{P_{0}} \phi_{\beta}\right\|+\left\|R_{P_{0}} L_{P_{0}} \phi_{\beta}-R_{P_{0}} L_{P_{0}} \phi\right\| \\
& +\left\|R_{P_{0}} L_{P_{0}} \phi-\phi\right\|<3 \varepsilon .
\end{aligned}
$$

by (5), (6) and (7). 
Remark. Clearly if a dual Banach space $E$ has the weak* normal structure then $E$ has the quasi-weak*-normal structure. But the converse is false. Indeed, let $E$ the space of absolutely summable real sequences with norm

$$
\|x\|=\max \left\{\left\|x^{+}\right\|_{1},\left\|x^{-}\right\|_{1}\right\}
$$

where $x^{+}, x^{-}$denote the positive and negative part of $x$ respectively and $\|x\|_{1}=\sum_{i=1}^{\infty}\left|x_{i}\right|$. Then, as shown by Lim [6] (Lemma 1 and Example 1), $E$ is a dual Banach space which does not have weak* normal structure. However, since $E$ is separable, an argument similar to that of Wong [13, Theorem 2] shows that $E$ has quasi-weak*-normal structure.

Problem 1. Does the trace class operator $\mathscr{T}(H)=\mathscr{C}(H)^{*}$ with dual norm have the weak* normal structure or the weak normal structure?

Lemma 3. Let $\Gamma$ be a non-empty set. Then $c_{0}(\Gamma)$ has the quasi-weaknormal structure if and only if $\Gamma$ is countable.

Proof. If $\Gamma$ is countable then $c_{0}(\Gamma)$ is norm separable. Hence each weakly compact convex subset of $c_{0}(\Gamma)$ has quasi-normal structure by Theorem 2 in [13].

Conversely, if $\Gamma$ is not countable, consider $\Gamma$ as a group (say the free group on $|\Gamma|$ generators). Pick $a \in \Gamma$. Let $f=\delta_{a}$ i.e. $f(x)=1$ if $x=a$ and $f(x)=0$ if $x \neq a$. Let $K$ denotes the closed convex hull of $\left\{l_{x} f ; x \in \Gamma\right\}$, where $\left(l_{x} f\right)(t)=f(x t), t \in \Gamma$. Then $K$ is weakly compact ([2, Corollary 3.7]) and $\operatorname{diam}(K)=1$. Now if $g \in K$, let $\sigma \subseteq \Gamma$ be a countable set such that $g(t)=0$ if $t \in \Gamma \sim \sigma$. Pick $z \in \Gamma \sim \sigma$ and let $h=\delta_{z}$. Then $h \in K$ and $\|g-h\|_{\infty}=1$. Hence $K$ does not have quasi-normal structure.

THEOREM 3. Let $H$ be a Hilbert space. Then $H$ is separable if and only if $\mathscr{C}(H)$ has quasi-weak-normal structure.

Proof. If $H$ is separable, then $\mathscr{C}(H)^{*}$ is separable [10, Proposition 2.1.10]). Hence $\mathscr{C}(H)$ is separable. Consequently every weakly compact convex subset of $\mathscr{C}(H)$ has quasi-normal structure by [13, Theorem 2].

Conversely, if $H$ is not separable, then $H$ is isomorphic to $l_{2}(\Gamma)$ for an uncountable set $\Gamma$. Consider the map $\rho: c_{0}(\Gamma) \rightarrow \mathscr{B}\left(l_{2}(\Gamma)\right)$ defined by

$$
\rho(f)(h)(t)=f(t) h(t), \quad t \in \Gamma,
$$

then $\rho$ is an isometry and an algebra isomorphism of $c_{0}(\Gamma)$ into $\mathscr{B}\left(l_{2}(\Gamma)\right)$. Furthermore, $\rho(f)$ is compact for each $f \in c_{0}(\Gamma)$. By Lemma 3, there exists a weakly compact convex subset $K$ in $c_{0}(\Gamma)$ which does not have 
quasi-normal structure. In particular, $\rho(K)$ is a weakly compact convex subset of $\mathscr{C}(H)$ which does not have quasi-normal structure also.

Summary. In the Table we shall abbreviate normal structure by n.s., quasi-normal structure by q.n.s., etc. We assume $\Gamma$ is not finite and $H$ is not finite dimensional.

\begin{tabular}{|c|c|c|}
\hline$\underline{c_{0}(\Gamma)}$ & $\underline{l_{1}(\Gamma)}$ & $\underline{l_{\infty}(\Gamma)}$ \\
\hline No n.s. & No n.s. & No n.s. \\
\hline q.w.n.s. & $\mathrm{w}^{*}$.n.s. & No q.w*.n.s \\
\hline$\Gamma$ is countable & w.n.s. & \\
\hline $\mathscr{C}(H)$ & $\mathscr{T}(H)$ & $\mathscr{B}(H)$ \\
\hline No n.s. & No n.s. & No n.s. \\
\hline q.w.n.s. & $\mathrm{w}^{*}$.n.s.(?) & No q.w*.n.s. \\
\hline$H$ is separable & w.n.s.(?) & \\
\hline & q.w*.n.s. & \\
\hline
\end{tabular}

3. On Lim's condition. Let $E$ be a Banach space. Then $E^{*}$ is said to satisfy Lim's condition if whether $\left\{\phi_{\alpha}\right\}$ is a bounded net in $E^{*}$, $\phi_{\alpha}$ converges to 0 in the weak* topology and $\lim _{\alpha}\left\|\phi_{\alpha}\right\|=s$, then $\lim _{\alpha}\left\|\phi_{\alpha}+\psi\right\|=s+\|\psi\|$ for any $\psi \in E^{*}$.

In [6], Lim showed that $l_{1}$ satisfies this condition for sequences.

LEMMA 4. Let $E$ be a Banach space. If $E^{*}$ satisfies Lim's condition, then $E^{*}$ has the following properties:

(a) Norm and weak ${ }^{*}$ topology agree on $S=\left\{\phi \in E^{*} ;\|\phi\|=1\right\}$

(b) For any $0<\varepsilon<2$, if $\left\{\phi_{\alpha}\right\}$ is a net in $E^{*},\left\|\phi_{\alpha}\right\| \leq 1, \phi_{\alpha} \rightarrow \phi$ in the weak $^{*}$-topology and $\left\|x_{\alpha}-x_{\beta}\right\| \geq \varepsilon$ for all $\alpha \neq \beta$, then $\|\phi\| \leq 1-\varepsilon / 2$.

In particular, $E^{*}$ has the Radon Nikodym Property and weak* normal structure.

Proof. (a) Let $\left\{\phi_{\alpha}\right\}$ be a net in $S, \phi \in S$ such that $\phi_{\alpha} \rightarrow \phi$ in the weak*-topology. Suppose $\left\|\phi_{\alpha}-\phi\right\| \rightarrow 0$. Then we may assume, by passing to a subnet if necessary, that $\left\|\phi_{\alpha}-\phi\right\| \geq \varepsilon$ for each $\alpha$. Since $\left\{\left\|\phi_{\alpha}-\phi\right\|\right\}$ is bounded by 2 , we may further assume that $\lim _{\alpha}\left\|\phi_{\alpha}-\phi\right\|=s \geq \varepsilon>0$. 
Let $\psi_{\alpha}=\phi_{\alpha}-\phi$. Then $\psi_{\alpha} \rightarrow 0$ in the weak*-topology but

$$
1=\lim _{\alpha}\left\|\phi+\left(\phi_{\alpha}-\phi\right)\right\|=\|\phi\|+s>1
$$

which is impossible.

(b) We may assume that $\left\|\phi_{\alpha}-\phi\right\| \geq \varepsilon / 2$ for each $\alpha$, and

$$
\lim _{\alpha}\left\|\phi_{\alpha}-\phi\right\|=s \text {. }
$$

Then by Lim's condition,

$$
\lim _{\alpha}\left\|\phi_{\alpha}\right\|=\lim _{\alpha}\left\|\left(\phi_{\alpha}-\phi\right)+\phi\right\|=s+\|\phi\|
$$

i.e. $s+\|\phi\| \leq 1$ or $\|\phi\| \leq 1-s \leq 1-\varepsilon / 2$.

The last statement follows from Corollary 8 and Proposition 9 in [8], and the proof of Theorem 3 [4] (That $E^{*}$ has weak* normal structure also follows simple modification of Lim's proof of Theorem 3 in [6]).

Given a locally compact Hausdorff space $X$, let $C_{0}(X)$ denote the $C^{*}$-algebra of complex-valued continuous functions $f$ on $X$ such that for any $\varepsilon>0$ there exists a compact subset $\sigma$ of $X$ such that $|f(x)| \leq \varepsilon$ for $x \in X \sim \sigma$ with the supremum norm.

THEOREM 4. Let $X$ be a locally compact Hausdorff space. The dual Banach space $C_{0}(X)^{*}$ satisfies Lim's condition if and only if $C_{0}(X)^{*}$ is isometric isomorphic to $l_{1}(\Gamma)$ for some non-empty set $\Gamma$.

Proof. If $C_{0}(X)^{*}$ satisfies Lim's condition, then, by Lemma 4, $C_{0}(X)^{*}$ has the Radon Nikodym Property. Since $C_{0}(X)^{* *}=M$ is the enveloping von Neumann algebra of the $C^{*}$-algebra $C_{0}(X)$, it follows from Theorem 4 in [3] that $M$ is the direct sum of Type $I$ factors i.e. $M$ is isomorphic to $\sum_{\alpha \in \Gamma} \oplus \mathscr{B}\left(H_{\alpha}\right)$. Since $M$ is commutative, $H_{\alpha}=C$ for each $\alpha \in \Gamma$. In particular, $C_{0}(X)^{*} \approx l_{1}(\Gamma)$.

Suppose $C_{0}(X)^{*}$ is isometric isomorphic to $l_{1}(\Gamma)$ for some non empty set $\Gamma$. We may assume that $\Gamma$ is infinite. Let $\left\{f_{\alpha}\right\}$ be a bounded net in $l_{1}(\Gamma)$ such that $f_{\alpha} \rightarrow 0$ in the weak*-topology and $\lim _{\alpha}\left\|f_{\alpha}\right\|=s$. Let $g \in l_{1}(\Gamma)$. Since $\left\|f_{\alpha}-g\right\| \leq\left\|f_{\alpha}\right\|+\|g\|$ for each $\alpha$, we may assume, by passing to a subnet if necessary, that $\lim _{\alpha}\left\|f_{\alpha}-g\right\|=t$ exists. Clearly we have $t \leq s+\|g\|$. To see that we actually have equality, let $\varepsilon>0$. Observe that in $l_{1}(\Gamma)$,

$$
\left\|f_{\alpha}-g\right\| \geq\left\|f_{\alpha}\right\|-\|g\|+2 \sum_{s \in \sigma}\left(|g(s)|-\left|f_{\alpha}(s)\right|\right)
$$


for any subset $\sigma$ of $\Sigma$. Now let $\sigma$ be a finite subset such that $\sum_{s \in \sigma}|g(s)|>$ $\|g\|-\varepsilon$. For this $\sigma$, we can choose $\alpha_{0}$, using the weak* convergence of $f_{\alpha}$ and the convergence of $\left\|f_{\alpha}\right\|$, so that for all $\alpha \geq \alpha_{0}$ we have $\sum_{s \in \sigma}\left|f_{\alpha}(s)\right|<\varepsilon$ and $\left\|f_{\alpha}\right\|>s-\varepsilon$. Then for all $\alpha \geq \alpha_{0}$ we have from (1)

$$
\left\|f_{\alpha}-g\right\| \geq s-\varepsilon-\|g\|+2\|g\|-2 \varepsilon-2 \varepsilon=s+\|g\|-5 \varepsilon .
$$

Thus $t \geq s+\|g\|$.

Problem 2. Let $X$ be a locally compact Hausdorff space. When does $C_{0}(X)$ * have the weak* normal structure?

THEOREM 5. Let $H$ be a Hilbert space. Then $\mathscr{T}(H)$ satisfies Lim's condition if and only if $H$ is finite dimensional.

Proof. If $H$ is finite dimensional, then $\mathscr{T}(H)$ is finite dimensional. Hence $\mathscr{T}(H)$ satisfies Lim's condition.

If $H$ is infinite dimensional, let $\left\{\xi_{n}, n=1,2, \ldots\right\}$ be an orthonormal sequence in $H$. For each $n=1,2, \ldots$, define $\phi_{n}(T)=\left\langle T \xi_{1}, \xi_{n}\right\rangle$. Then $\phi_{n} \in \mathscr{T}(H),\left\|\phi_{n}\right\|=1$ and $\phi_{n} \rightarrow 0$ weakly. Indeed, if $T \in \mathscr{B}(H)$, then

$$
\infty>\left\|T \xi_{1}\right\|^{2}=\sum_{\alpha \in I}^{\infty}\left|\left\langle T \xi_{1}, \xi_{\alpha}\right\rangle\right|^{2} \geq \sum_{n=1}^{\infty}\left|\left\langle T \xi_{1}, \xi_{n}\right\rangle\right|^{2}=\sum_{n=1}^{\infty}\left|\phi_{n}(T)\right|^{2}
$$

where $\left\{\xi_{\alpha}\right\}_{\alpha \in I}$ is a complete orthonormal set of $H$ containing $\left\{\xi_{n}\right\}$. So $\phi_{n}(T) \rightarrow 0$. Also $\left\|\phi_{n}-\phi_{1}\right\| \leq \sqrt{2}$ for each $n$. Hence $\varlimsup_{n}\left\|\phi_{n}-\phi_{1}\right\| \leq \sqrt{2}$ i.e. $\lim _{n}\left\|\phi_{n}-\phi_{1}\right\| \neq \lim \left\|\phi_{n}\right\|+\left\|\phi_{1}\right\|$. In particular, $\mathscr{T}(H)$ does not satisfy Lim's condition.

\section{REFERENCES}

[1] M. S. Brodskii and D. P. Milman, On the center of a convex set, Dok1. Akad. Nauk SSSR, 59 (1948), 837-840 (Russian).

[2] R. B. Burkel, Weakly Almost Periodic Functions on Semigroups, Gordon and Breach (1970).

[3] C. C. Chu, A note on scattered $C^{*}$-algebras and the Radon-Nikodym property, J. London Math. Soc., 24 (1981), 533-536.

[4] D. van Dulst and Brailey Sims. Fixed points of nonexpansive mappings and Chebyshev centers in Banach spaces with norms of type ( $K K)$. Preprint.

[5] E. G. Effros, Order ideals in a $C^{*}$-algebra and its dual, Duke Math. J., 30 (1963), 391-412.

[6] T. C. Lim, Asymptotic centers and nonexpansive mappings in conjugate spaces, Pacific J. Math., 90 (1980), 135-143.

[7] K. McKennon, Multipliers, positive functionals, positive-definite functions, and Fourier-Steiltjes transforms, Memoir Amer. Math. Soc., 111 (1971). 
[8] I. Namioka and R. R. Phelps, Banach spaces which are Asplund spaces, Duke Math. J., 42 (1975), 735-750.

[9] D. Roux and C. Zanco, Kannan maps in normed spaces, Rend. Sc. fis. mat. e nat, LXV (1978), 252-258.

[10] S. Sakai, $C^{*}$-algebras and $W^{*}$-algebras, Springer-Verlag (1971).

[11] P. M. Soardi, Struttura quasi normale e teoremi di punto unito, Rend. 1st Mat. Univ. Trieste 4 (1972), 105-114.

[12] M. Takesaki, Theory of Operator Algebras I, Springer-Verlag 1979.

[13] C. S. Wong, Close-to-normal structure and its applications, J. Funct. Anal., 16 (1974), 353-358.

Received May 11, 1984. This research is supported by NSERC Grant A7679 and A8065.

UNIVERSITY OF AlbERTA

EDMonton, Alberta T6H 5M3

CANADA

AND

LAKEHEAD UNIVERSITY

THUNDERBAY, ONTARIO P7B 5E1

CANADA 


\section{PACIFIC JOURNAL OF MATHEMATICS EDITORS}

V. S. VARADARAJAN (Managing Editor)
University of California
Los Angeles, CA 90024
HEBERT CLEMENS
University of Utah
Salt Lake City, UT 84112
CHARLES R. DEPRIMA
California Institute of Technology
Pasadena, CA 91125

\section{R. FINN}

Stanford University

Stanford, CA 94305

HeRmanN FlaschKa

University of Arizona

Tucson, AZ 85721

RAMESH A. GANGOLli

University of Washington

Seattle, WA 98195

ROBION KIRBY

University of California

Berkeley, CA 94720

\section{C. MoOre}

University of California

Berkeley, CA 94720

H. SAMELSON

Stanford University

Stanford, CA 94305

HAROLD STARK

University of California, San Diego

La Jolla, CA 92093

ASSOCIATE EDITORS
R. ARENS
E. F. BECKENBACH
B. H. NeUmanN
F. WOLF
K. Yoshida (1906-1982)

\section{SUPPORTING INSTITUTIONS}

UNIVERSITY OF ARIZONA

UNIVERSITY OF BRITISH COLUMBIA

CALIFORNIA INSTITUTE OF TECHNOLOGY

UNIVERSITY OF CALIFORNIA

MONTANA STATE UNIVERSITY

UNIVERSITY OF NEVADA, RENO

NEW MEXICO STATE UNIVERSITY

OREGON STATE UNIVERSITY
UNIVERSITY OF OREGON

UNIVERSITY OF SOUTHERN CALIFORNIA

STANFORD UNIVERSITY

UNIVERSITY OF HAWAII

UNIVERSITY OF TOKYO

UNIVERSITY OF UTAH

WASHINGTON STATE UNIVERSITY

UNIVERSITY OF WASHINGTON 


\section{Pacific Journal of Mathematics}

\section{Vol. 121, No. $1 \quad$ November, 1986}

Om P. Agrawal, Douglas Napier Clark and Ronald George Douglas,

Invariant subspaces in the polydisk $\ldots \ldots \ldots \ldots \ldots \ldots \ldots \ldots \ldots \ldots$

Christoph Bandt and Gebreselassie Baraki, Metrically invariant measures

on locally homogeneous spaces and hyperspaces $\ldots \ldots \ldots \ldots \ldots \ldots \ldots$

Marcy Mason Barge, Horseshoe maps and inverse limits ..............29

Russell Gene Bilyeu, Robert Richard Kallman and Paul Weldon Lewis,

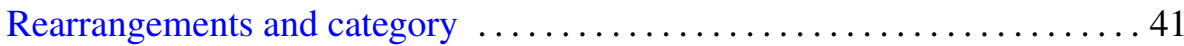

Jean Bourgain, A problem of Douglas and Rudin on factorization . . .......47

Hernan Cendra, A normal form and integration in finite terms for a class of

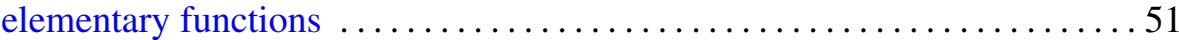

Ky Fan, The angular derivative of an operator-valued analytic function . . . . 67

Gerhard Gierz, On the Dunford-Pettis property of function modules of

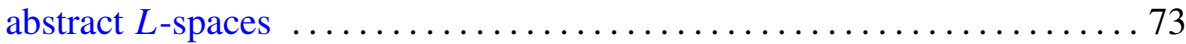

Gabriel Katz, On polynomial generators in the algebra of complex functions



Ridgley Lange, Duality and asymptotic spectral decompositions $\ldots \ldots \ldots . .93$

Anthony To-Ming Lau and Peter F. Mah, Quasinormal structures for certain spaces of operators on a Hilbert space ................... 109

R. Daniel Mauldin, Correction: "The set of continuous nowhere differentiable functions"

Alan Harvey Mekler and Saharon Shelah, $\omega$-elongations and Crawley's problem

Alan Harvey Mekler and Saharon Shelah, The solution to Crawley's problem

Richard Rochberg, Deformation of uniform algebras on Riemann surfaces

Joseph Roitberg, On weak epimorphisms in homotopy theory

Jesús M. Ruiz, A remark on fields with the dense orbits property

Henry Wente, Counterexample to a conjecture of H. Hopf

David G. Wright, Rigid sets in $E^{n}$ 